\title{
How much impact do antimicrobial surfaces really have on healthcare-acquired infection?
}

Comment on CID article, 'Impact of a Novel Antimicrobial Surface Coating on HealthcareAssociated Infections and Environmental Bioburden at Two Urban Hospitals'

Stephanie J. Dancer MD, FRCPath ${ }^{1,2^{*}}$

\section{Affiliation}

1. Dept. of Microbiology, Hairmyres Hospital, NHS Lanarkshire, Scotland, UK

2. School of Applied Sciences, Edinburgh Napier University, Scotland, UK

\section{Corresponding author*}

Professor S. J. Dancer, Dept. of Microbiology, Hairmyres Hospital, Lanarkshire G75 8RG

E-mail: stephanie.dancer@lanarkshire.scot.nhs.uk

Tel: +44-1355 584792 
Interest remains in the origins of healthcare-associated infection (HAI). If we knew how many infections originated from hands; environment; equipment; air; visitors; or patients themselves, then infection prevention activities could be prioritised according to risk. The paper from Ellingson et al chooses to focus on the role of the environment by applying a quaternary ammonium organosilane to surfaces in two different hospitals. ${ }^{1}$ It describes the clinical and environmental impact of this coating in a medical ICU and two medical wards in Hospital A; and medical and neurological ICUs and transplant step-down unit in Hospital B. While there were notable differences between the two hospitals, the results suggest that surface bioburden, including pathogens, was significantly reduced, along with decreased rates of multidrug resistant organism (MDRO) bloodstream infections (BSI) and Clostridium difficile infections (CDI).

At first sight, this seems like the study we have been waiting for, to seal the role of the environment in HAI. Healthcare surfaces serve as a reservoir for potential pathogens, which are able to survive for weeks in temperate surroundings. ${ }^{2}$ Pathogens may be controlled by cleaning and decontamination practices but these do not necessarily eliminate all risk. It is fair to say that replacing surfaces with antimicrobial products might offer an additional safeguard when there are cleaning deficits. Such coatings provide an alternative to the daily rigours of cleaning and could even temper the debate over how this should be done. ${ }^{3}$ But life is not that simple and delving into study methods uncovers some apposite concerns over missing detail and interpretation. To begin with, the coating is billed as 'bactericidal', when we know that many other microorganisms adhere to surfaces, namely spores, fungi and viruses. Quaternary ammonium compounds are generally inactive against CD spores; ${ }^{4}$ so how does this coating decrease surface contamination and infection rates of C.difficile? The authors suggest that the coating generates an electric charge, which encourages adhesion potential for spores and makes them less likely to be aerosolized or transferred to other surfaces. ${ }^{5}$ This may well be the case but we need robust experimental investigation for all the relevant pathogens, including spores, which were absent from the reference provided.

Is the coating active against the new-kid-on-the-block, Candida auris? ${ }^{6}$ There were clear reductions in surface bacterial counts but no data on fungi. Since Trypticase Soy Agar supports fungal growth, should we assume that the counts were based on environmental bacteria, with fungal colonies excluded? This would have been technically difficult to perform so perhaps fungal colonies were indeed part of the overall surface count. There was no detail on the fungal contribution to MDRO-BSI data.

However effective the coating might be on dispelling bioburden, there was still a necessity for it to be replenished at regular intervals throughout the study. A shelf life of 15 weeks is a very short time in the life of a hospital. Estates departments may not have the resources to be able to spray all selected sites in clinical areas every four months or so. Study sponsors would have absorbed the costs of the product, application and re-application for the study itself but there was no indication of initial and ongoing costs of neither the product nor its 
application for interested parties to compare. Infection control personnel (and managers) need to know that interventions provide cost-benefit.

Such coatings are ineffective if applied to surfaces without prior cleaning and this was clearly described in the study. However, there were no assurances over any physical effects from the coating or its reapplication, such as discolouration of treated surfaces, or changes in texture, such as grittiness or stickiness from product build up. ${ }^{7}$ We also need to know whether the coating interacts with other fluids used in the healthcare environment, particularly for decontamination. There are many powerful disinfectants on the market, the components of which might react with the product to release substances that could be harmful. They may inactivate the coating or even strip it from its host surface. The product requires independent verification of activity against a full range of microbes as well as data on chemical composition and reaction following exposure to other compounds.

What is the risk that environmental flora, including pathogens, could become tolerant to quaternary ammonium compounds during long term exposure? ${ }^{8}$ This is particularly pertinent when the same, or similar, chemicals are also being used as disinfectants for routine cleaning. Sustained effects of the product, and indeed, any others that contain antimicrobial agents must be elucidated before widespread adoption in healthcare environments. The concern is that organisms evolve survival mechanisms when habitually exposed to a substance that could potentially damage or destroy them; these mechanisms may include the facility for antimicrobial resistance that could be transmitted to other environmental flora, including pathogens. ${ }^{8}$

The study was not randomised so staff (and patients) would have known about the intervention and domestic staff would have witnessed the sampling procedures. Given that cleaners always react to environmental monitoring, they may have altered their delivery in order to preclude criticism. ${ }^{3}$ There might even have been the opposite effect, that of diminution of diligent cleaning if staff knew that the coating was capable of eliminating pathogens! The study design did not audit cleaning practices and therein illustrates a classic confounder to any study investigating environmental decontamination.

Of course, cleaners are not the only staff segment that may have changed their practices in response to this study. There would have been other personnel in the vicinity whose behaviour might have been affected by application of an antimicrobial coating. This includes study personnel themselves, especially as there remains concern over industry involvement in healthcare research; bias is difficult to ignore and not always detectable. ${ }^{9}$ There is some sympathy towards companies trying to obtain recognition for new products but widespread acceptance should, and must, depend on quantity and quality of independent studies.

While mentioned, no antimicrobial consumption data was presented and this represents another major confounder. Changes in antimicrobial prescribing could have facilitated most, if not all, the data presented in this paper. ${ }^{10}$ There were also inferences of changes in 
isolation policies and hand hygiene compliance, but nothing on outbreak occurrence, either before or during the study. Could the reduction in CDI, in particular, represent reversion to the norm following increased incidence? Or, was the lack of effect in Hospital B due to undetected cross-infection? It would have been helpful to have seen an appendix containing detail on MDRO-BSI for both hospitals.

Definitions of BSI do not always correctly identify 'infection'; nor presence of quoted organism in the blood stream (e.g. contamination during collection or processing); nor legitimate healthcare contribution (e.g. present but undetected or masked before hospital admission). ${ }^{11}$ MDROs themselves encompass microorganisms that originate from the healthcare environment, i.e. the proportion that might be repelled by an antimicrobial coating, but there are others that come from endogenous reservoirs such as skin, mucous membranes and gastrointestinal system. ${ }^{12}$ These are independent of any impact elicited by an antimicrobial surface coating. Indeed, direct transmission of MDROs from the air; or from another patient or uncoated surface, such as ward items or equipment; laundry; visitors; consumables and gifts, would bypass any generic coating of selected surfaces. This then begs the question, are the data presented in this paper biologically plausible?

Six years ago a study was published suggesting that copper coated surfaces could halve the rate of HAI. ${ }^{13}$ The study outcomes were complex, the reporting intentionally selective, and a final evaluation that contradicted biological plausibility. ${ }^{14}$ According to Weinstein, up to $60 \%$ acquired infection is endogenous, with $20 \%$ attributed to staff hands and another $20 \%$ originating from exogenous sources, including environment. ${ }^{12}$ This proportion is supported by a recent study using whole genome sequencing to track one pathogen (Staphylococcus aureus) in an ICU. ${ }^{15}$ Systematic screening of patients, surfaces, air and staff hands found that 59\% transmission events occurred between colonised patients and their own body sites, i.e. endogenous transmission, including BSIs. There were $30 \%$ transmission events involving the environment, including air, but only $12 \%$ that linked patients directly with hand-touch sites.

In short, the copper study conclusion was contested because the magnitude of reduction assumed to relate directly to the environment was not biologically feasible. ${ }^{14}$ Now we have a similar study, suggesting that chosen HAI indicators (MDRO BSIs and CDI) decreased by over a third after some, but not all, surfaces were coated with an antimicrobial agent. Are surface pathogens ultimately responsible for more than a third invasive HAls? How many environmental pathogens find their way into a patient after a minimum of 2 days in hospital, specifically gut and blood stream? ${ }^{12,16,17}$

We should put it to the authors that their findings are biologically implausible, given the detail supplied. But, we remain intrigued; hopeful, and mindful of future studies, designed to test the aims expounded in this paper. It is not impossible that an antimicrobial coating of some type will become standard for all healthcare surfaces one day. One wonders what our domestic staff will do then. 


\section{Declarations of interest}

The author has no potential conflicts of interest.

\section{Funding}

No funding was required for the writing of this article.

\section{References}

1. Ellingson KD, Pogreba-Brown k, Gerba C, Elliott SP. Impact of a novel antimicrobial surface coating on healthcare-associated infections and environmental bioburden at two urban hospitals. Clin Infect Dis 2019, in press.

2. Dancer SJ. The role of hospital cleaning in the control of hospital-acquired infection. $J$ Hosp Infect 2009; 73: 378-85.

3. Dancer SJ. Do's and don'ts for hospital cleaning. Curr Opin Infect Dis 2016; 29(4): 415-23.

4. Fraise A. Currently available sporicides for use in healthcare, and their limitations. $J$ Hosp Infect 2011; 77: 210-212.

5. Kugler R, Bouloussa O, Rondelez F. 2005. Evidence of a charge-density threshold for optimum efficiency of biocidal cationic surfaces. Microbiology 205; 151:1341-8.

6. Chowdhary A, Voss A, Meis JF. Multidrug-resistant Candida auris: 'new kid on the block' in hospital-associated infections? J Hosp Infect 2016; 94(3):209-212.

7. Ramsden JJ, Reid M, Whatley V, Dancer S.J. Disastrous performance of NanoCote/Aqua-Based antimicrobial paint in a hospital setting. J Biolog Physics Chem 2016; 16(3): 131-136.

8. Maillard JY. Resistance of bacteria to biocides. Microbiol Spectr 2018; 6(2). doi: 10.1128/microbiolspec.ARBA-0006-2017.

9. De Kraker M, Harbarth S, Dancer SJ. Shining a light on ultraviolet-C disinfection: no golden promises for infection prevention. Am J Infect Control 2018; 46(12):14221423.

10. Dancer SJ, Kirkpatrick P, Corcoran DS, Christison F, Farmer D, Robertson C. Approaching zero: temporal effects of a restrictive antibiotic policy on hospitalacquired Clostridium difficile, extended-spectrum $\beta$-lactamase-producing coliforms and meticillin-resistant Staphylococcus aureus. Int J Antimicrob Agents 2013; 41: 137-142.

11. Seigel TA, Cocchi MN, Salciccioli J, Shapiro NI, Howell M, Tang A, Donnino MW. Inadequacy of temperature and white blood cell count in predicting bacteremia in patients with suspected infection. J Emerg Med 2012; 42(3): 254-9. 
12. Weinstein, RA. Epidemiology and control of nosocomial infections in adult intensive care units. Am J Med 1991; 91: 179S-184S.

13. Salgado CD, Sepkowitz KA, John JF, Cantey JR, Attaway HH, Freeman KD, Sharpe PA, Michels HT, Schmidt MG. Copper surfaces reduce the rate of healthcare-acquired infections in the intensive care unit. Infect Control Hosp Epidemiol 2013; 34(5):47986.

14. Harbarth S, Maiwald M, Dancer SJ. The environment and healthcare-acquired infections: why accurate reporting and evaluation of biological plausibility are important. Infect Control Hosp Epidemiol 2013; 34(9): 996-7.

15. Dancer SJ, Adams C, Smith J, Pichon B, Kearns A, Morrison D. Tracking Staphylococcus aureus in the intensive care unit using whole-genome sequencing. $J$ Hosp Infect 2019; 103(1):13-20.

16. Grundmann H, Bärwolff, S, Tami, A, et al. How many infections are caused by patient-to-patient transmission in intensive care units? Crit Care Med 2005; 33: 946951.

17. Kola A, Schwab F, Bärwolff $S$, et al. Is there an association between nosocomial infection rates and bacterial cross transmissions? Crit Care Med 2009; 38: 46-50. 